\title{
Effect of astaxanthin on cutaneous wound healing
}

\author{
Jitlada Meephansan' \\ Atiya Rungjang' \\ Werayut Yingmema ${ }^{2}$ \\ Raksawan Deenonpoe ${ }^{3}$ \\ Saranyoo Ponnikorn ${ }^{3}$ \\ 'Division of Dermatology, Chulabhorn \\ International College of Medicine, \\ Thammasat University, Pathum Thani, \\ Thailand; ' 2 Laboratory Animal Centers, \\ Thammasat University, Pathum Thani, \\ Thailand; ${ }^{3}$ Chulabhorn International \\ College of Medicine, Thammasat \\ University, Pathum Thani, Thailand
}

Correspondence: Jitlada Meephansan Division of Dermatology, Chulabhorn International College of Medicine, Thammasat University, Rangsit Campus, 99 Moo 18 Phahonyothin Road, Klongluang, Pathum Thani, I2I20, Thailand

Tel +66 25644444 ext I535

Email kae_mdcu@yahoo.com
This article was published in the following Dove Press journal:

Clinical, Cosmetic and Investigational Dermatology

13 July 2017

Number of times this article has been viewed
Abstract: Wound healing consists of a complex series of convoluted processes which involve renewal of the skin after injury. ROS are involved in all phases of wound healing. A balance between oxidative and antioxidative forces is necessary for a favorable healing outcome. Astaxanthin, a member of the xanthophyll group, is considered a powerful antioxidant. In this study, we investigated the effect of topical astaxanthin on cutaneous wound healing. Full-thickness dermal wounds were created in 36 healthy female mice, which were divided into a control group and a group receiving $78.9 \mu \mathrm{M}$ topical astaxanthin treatment twice daily for 15 days. Astaxanthin-treated wounds showed noticeable contraction by day 3 of treatment and complete wound closure by day 9 , whereas the wounds of control mice revealed only partial epithelialization and still carried scabs. Wound healing biological markers including Col1A1 and bFGF were significantly increased in the astaxanthin-treated group since day 1 . Interestingly, the oxidative stress marker iNOS showed a significantly lower expression in the study. The results indicate that astaxanthin is an effective compound for accelerating wound healing.

Keywords: astaxanthin, wound healing, reactive oxygen species, antioxidant

\section{Introduction}

Wound healing or repair is a complex and crucial process of response to injury. To accomplish this, coordination of multiple cells and components is necessary. The healing process is composed of three overlapping phases: inflammation, proliferation, and remodeling. ${ }^{1}$ In the coagulation and inflammatory phase, cutaneous injury affecting primarily the epithelial and endothelial compartments results in a coagulation cascade forming a blood clot and release of pro-inflammatory mediators. The blood clot within the vessel lumen provides hemostasis, and the clot within the injury site acts as a provisional matrix for cell migration, promoting formation of fresh extracellular matrix (ECM), a reservoir for cytokines and growth factors. Inflammatory white cell functions include debridement of necrotic material and bacteria, and production of critical cytokines. Twenty-four to forty-eight hours after injury, monocytes replace neutrophils and differentiate into tissue macrophages which phagocytose and kill bacteria, scavenge tissue debris, and release several growth factors. The growth factors stimulate migration and proliferation of fibroblasts, endothelial cells, and keratinocytes, and production and modulation of ECM, constituting the proliferation/migration phase resulting in reepithelialization and angiogenesis. The remodeling phase begins 5-7 days after injury to break down excess macromolecules. Cells within the wound are returned to a stable phenotype and ECM material is altered. 
ROS are involved in all phases of wound healing. ROS are small chemically reactive molecules of oxygen such as oxygenfree radicals. They strongly react with multiple molecular components such as nucleic acids, proteins, lipids, and other small inorganic molecules. ROS may alter the function or irreversibly destroy the target molecule through their cascading reactions. On the other hand, low concentrations of ROS play a role in initiating signaling during the proliferative phase, including homeostasis, ${ }^{2,3}$ formation of granulation tissue, reepithelialization, and angiogenesis. ${ }^{4}$ The physiological level of ROS, such as hydrogen peroxide at the wound site, increases after injury and gradually declines. Prolonged generation or large amounts of ROS, called oxidative stress, can cause chronic inflammation by initiating the NF-kB/Rel pathway, ${ }^{4}$ which is believed to be the major cause of chronic unhealed wounds. The negative effects of oxidative stress can be restrained by antioxidant enzyme systems and dietary antioxidants such as carotenoids. ${ }^{5}$ The carotenoid structure has a common chemical feature containing a long-conjugated double-bond polyene chain, which has an ability to quench or scavenge ROS. ${ }^{6}$ Several kinds of antioxidants are proposed to regulate the oxidation-reduction balance, and studies of the effects of various antioxidants on wound healing have reported accelerated healing outcomes. ${ }^{7,8}$

Astaxanthin, a member of the xanthophyll group, is a red-orange carotenoid. Its antioxidative effect has been shown to exceed those of pro-vitamin A and vitamin E, ${ }^{9}$ and it is considered one of the most powerful antioxidants. The interest in antioxidant activity of astaxanthin in the pharmaceutical industry, aquaculture, and nutritional health is expanding. It has been used as a highly effective antioxidant in various health conditions. ${ }^{10}$ In dermatology, clinical studies suggest that oral supplementation and topical treatment of an astaxanthin extract from Haematococcus pluvialis improves the skin condition and provides protective effects mediated by balanced oxidative actions. A balance between oxidative and antioxidative forces is needed for favorable wound healing. Among studies on various health-promoting effects of astaxanthin, very few studies on wound healing have been reported. In this study, we investigated the effect of topical astaxanthin on cutaneous wound healing in animal models, serving as a preliminary study for the use of astaxanthin in accelerating wound healing. We evaluated wound contraction area and histopathology, and determined the mRNA levels of iNOS, Col1A1, and bFGF at the wound area.

\section{Materials}

\section{Drugs}

The astaxanthin material, composed of $78.9 \mu \mathrm{M}$ of astaxanthin extracted from $H$. pluvialis, was supplied by China
Jiangsu International Economic and Technical Cooperation Group, Ltd. The vehicle was palm oil.

\section{Animals}

The animal protocol (no 015/2558) was approved by the Institutional Animal Care and Use Committee of Thammasat University, which is accredited by the National Research Council of Thailand. All animals were housed at the Laboratory Animal Center of Thammasat University according to guidelines for the care and use of laboratory animals, National Research Council 2011. Young female BALB/c mice ( 8 weeks old) were procured from the National Laboratory Animal Center, Thailand. A total of 36 mice were randomly assigned to an astaxanthin-treated group and a control group. During the experiments, the animals were housed under strict hygiene standards and controlled environmental conditions (12-hour light/dark cycle, temperature approximately $23^{\circ} \mathrm{C}$ ). Standard laboratory food and water were provided ad libitum.

\section{Animal experiments}

\section{Anesthesia and surgical procedure}

Mice were anesthetized with $1.5 \%$ isoflurane in $100 \%$ oxygen at a $0.9 \mathrm{~L} /$ minute flow after induction with $5 \%$ isoflurane using a single circuit anesthesia system. Mice were positioned in ventral recumbency. Hair on the dorsal surface of the skin was removed with a razor, the skin was aseptically prepped with $70 \%$ alcohol gauze sponges, and a sterile drape positioned. All wounding procedures were performed under sterile conditions by one surgeon. The dorsal skin was picked up and a punch hole extending through the panniculus carnosus was made using a 4-mm sterile disposable biopsy punch. Two full-thickness wounds were created at designated locations. The wounds were left open with no dressing. Each wound site was digitally photographed and tissues were collected. Tissues at wound sites were collected for reverse transcription polymerase chain reaction (RT-PCR) on days $1,3,6,9,12$, and 15 using a disposable biopsy punch (6 mm in diameter).

Tissues for histopathology were collected from treatment and control groups by elliptical excision on day 3 and 7 post-wounding.

\section{Post-operative and wound care}

The wounds were treated topically twice daily with astaxanthin extract in the treatment group and vehicle in control group ( $0.025 \mathrm{~mL} /$ wound). A digital image of each wound with a scale was recorded daily until complete closure. For the wound 
contraction study, a template containing a 10-mm diameter circular window was used to standardize the size of each wound.

\section{Outcome measures}

\section{Wound contraction}

Digital photographs were taken on the day of surgery and every day from then on. Time to wound closure was defined as the time point at which the wound bed was filled with new tissue. Wound area was analyzed by tracing the wound margin with a fine-resolution computer mouse and calculating the pixel area using Adobe Illustrator CS6 software, run on an Intel ${ }^{\circledR}$ Core $^{\mathrm{TM}}$ i7-2600K CPU. All tracing was performed by the same computer graphics professional, without knowledge of treatment conditions. Wound contraction was calculated as the percentage decrease in original wound area. Complete closure was considered when the wound area disappeared, and became grossly equal to zero.

\section{RT-PCR}

Collected tissue samples were stored fresh frozen at $-80^{\circ} \mathrm{C}$ until use. The RNeasy Mini Kit (Qiagen NV, Venlo, the Netherlands) was used for extraction and purification of total RNA from tissues, following the manufacturer's protocol. A260/280 ratios of the samples were used to evaluate the RNA quantity and quality. cDNA synthesis was performed using the ImProm-IITM Reverse Transcription System (Promega Corporation, Fitchburg, WI, USA) following the manufacturer's protocol. Expression of the wound healing markers Col1A1 (Mm00801666_g1), bFGF (Mm00438930_m1), and iNOS (qMmuCIP0035502) was analyzed using realtime PCR following the manufacturer's protocol from iTaq Universal Probes Supermix (Bio-Rad Laboratories Inc., Hercules, CA, USA). The relative ratio of gene expression for each gene was determined by standard exponential curves using the CFX 96 Touch $^{\mathrm{TM}}$ PCR Detection System (BioRad Laboratories Inc.). The internal control gene (B2M, Hs00985689_m1; Thermo Fisher Scientific, Waltham, MA, USA) was used to normalize target gene expression.

\section{Histopathological evaluation}

Histologic evaluation was performed using visible light microscopy. The samples were fixed in $10 \%$ buffered formalin. After fixation, sections perpendicular to the anteriorposterior axis of the wound were dehydrated with graded ethanol and embedded in paraffin. Hematoxylin and eosin, and Masson's trichrome stains were used on sections of paraffin-embedded tissue. Images were captured at $4 \times$ and $10 \times$ magnification with a Leica DM3000 LED microscope under the same exposure. Reepithelialization, granulation tissue formation, angiogenesis, and inflammatory cell infiltration were evaluated.

\section{Statistical analysis}

Results were recorded as mean \pm SD. The Student's $t$-test and Mann-Whitney test were performed to analyze differences between data obtained from different experimental groups. A $P$-value of less than 0.05 was considered significant.

\section{Results \\ Wound contraction}

The astaxanthin extract applied topically on the wounds showed significant acceleration of wound closure which was clearly visible at day 3 of the experiment (Figure 1). On day 9 after wounding, the astaxanthin-treated wounds had already lost their eschars and appeared fully epithelialized, whereas the wounds of control mice showed only partial epithelialization and still carried scabs. Complete wound closure in the control group was observed only by day 11 . The mean original wound area in the astaxanthin-treated group was larger than that in the control group, at $14.69 \mathrm{~mm}^{2}$ and $12.42 \mathrm{~mm}^{2}$, respectively. In addition, on day 1 post-injury, wound area in the astaxanthin-treated group significantly decreased to $10.33 \mathrm{~mm}^{2}$ ( $28.15 \%$ reduction of the original wound area), whereas the control group showed an $18.12 \%$ reduction, to $10.23 \mathrm{~mm}^{2}$. On day 7 after wounding, closure of the wounds in the astaxanthin-treated group was more pronounced than that in the control group, at $90 \%\left(1.27 \mathrm{~mm}^{2}\right)$

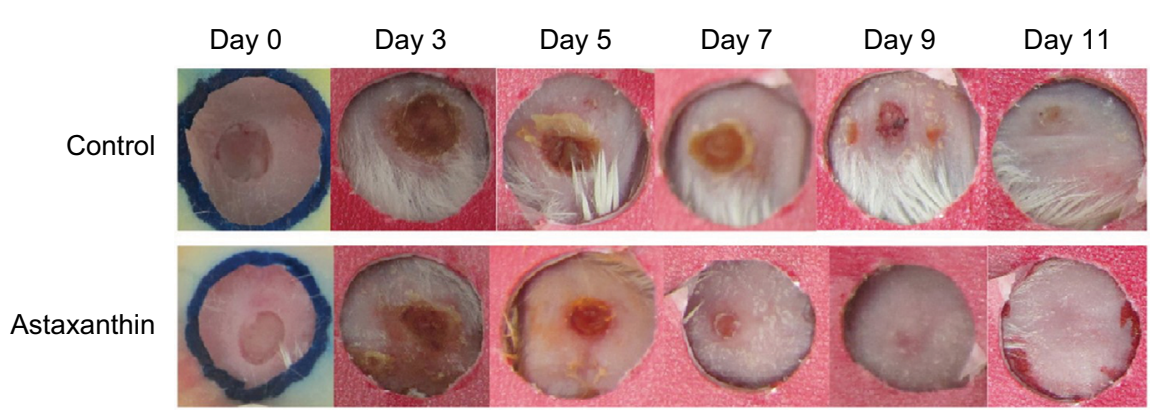

Figure I Images of wound contraction in astaxanthin and control groups from day 0 to day II. 
and $82.35 \%\left(2.17 \mathrm{~mm}^{2}\right)$, respectively (Figure 2$)$. No mice were excluded from the study and no wounds showed signs of infection. At the end of the experiment, mice in both groups showed complete wound closure without visible scars or chronic wounds.

The data indicated that wound closure in astaxanthin-treated mice was significantly accelerated compared with vehicletreated mice. A significant difference between the two groups was observed beginning at day 1 and continued until complete wound closure in the astaxanthin-treated group at day 10 .

\section{Histology}

At day 3, almost complete reepithelialization was observed in the astaxanthin-treated group but poor reepithelialization was seen in the control group. In the control group, stellate and spindle fibroblasts were scattered in the granulation tissue with a moderate to high degree of edema, whereas collagen bundles with a mild degree of edema were observed in the astaxanthintreated group. In addition, a few capillary vessels were present in the wound area which were poorly arranged in the control group, but were well arranged in the astaxanthin-treated group. Mononuclear cells, which were marginated in the vessels and scattered in perivascular and dermis regions, were markedly decreased in the astaxanthin-treated group compared to the control group. At day 7, complete reepithelialization and a wellelongated epidermis with keratinization was observed in mice treated with astaxanthin. The degree of infiltrated inflammatory cells was minimal in both groups. Well-formed granulation tissue, fibroblasts oriented parallel to the skin surface, and abundant organization of collagen were observed in the astaxanthintreated group. Treatment with astaxanthin significantly promoted the wound healing process in mice (Figure 3).

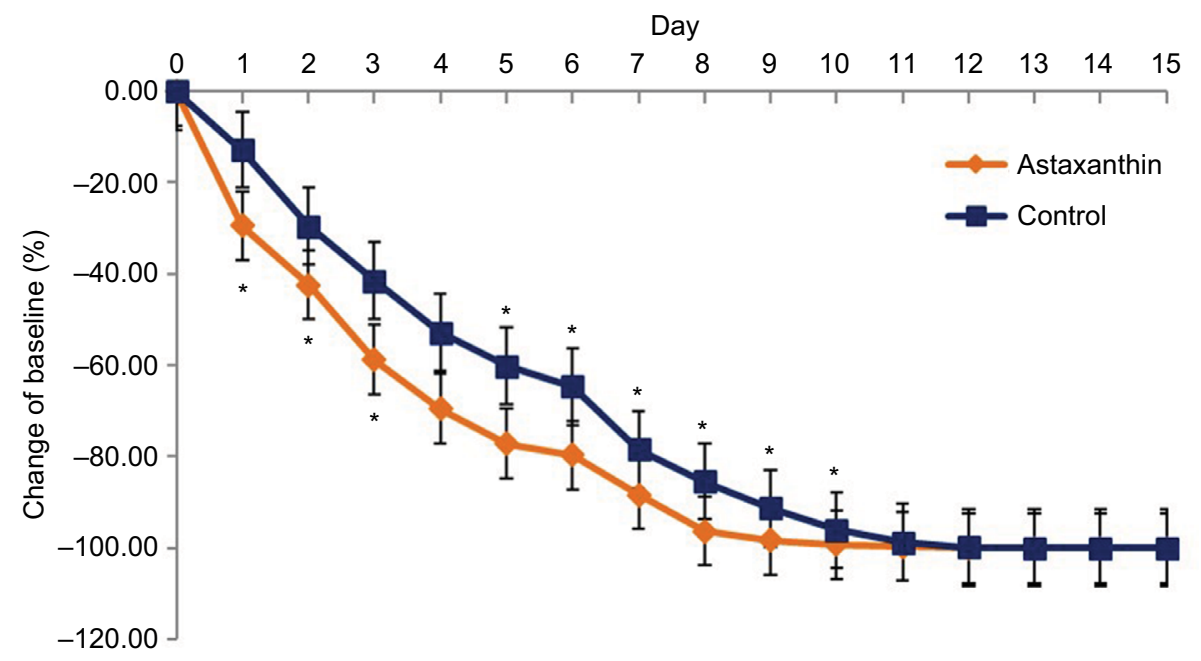

Figure 2 Wound area contraction in astaxanthin and control groups from day 0 to day 15 . Notes: Error bars indicate standard deviations. $* P<0.05$.

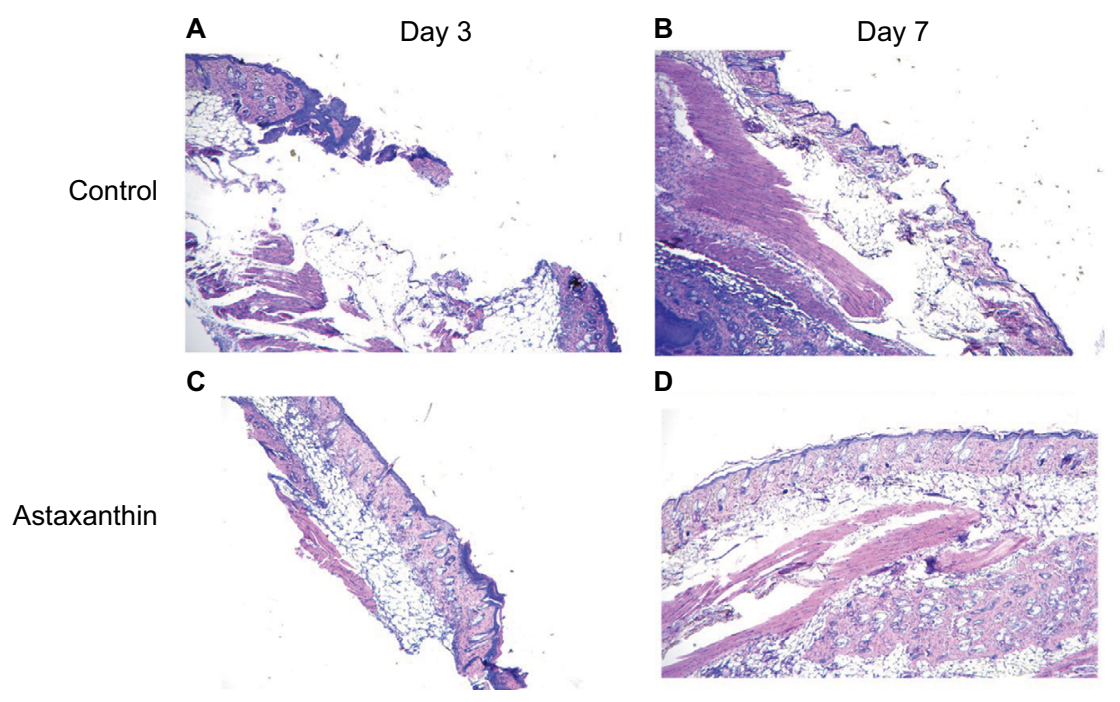

Figure 3 Skin wound sections from the astaxanthin-treated group and the control group at day 3 and day 7 stained with hematoxylin and eosin. Notes: (A, B) Control; (C, D) astaxanthin-treated group; (A, C) and (B, D) represent day 3 and day 7 of the experiment, respectively. 


\section{Wound healing markers and oxidative stress markers}

The mRNA expression of tissue-specific wound healing biomarkers and oxidative stress biomarkers including Col1A1, bFGF, and iNOS were determined using real-time PCR. Dynamic gene expression profiles in astaxanthin-treated mice and the control group were compared on days 1-15 (Figure 4). During the healing process, expression of CollA1 and bFGF in the astaxanthin-treated group was higher than that in the control group, throughout the healing period. The expression of collagen1 mRNA in both groups was markedly increased and reached a maximum on day 6 post-injury. bFGF mRNA expression in both groups decreased during the first 3 days post-injury, and then gradually increased until day 15 in both groups. iNOS was selected as an oxidative stress marker, for monitoring ROS during the wound healing process. Real-time PCR analysis revealed a decrease in expression of iNOS in astaxanthin-treated mice from day 3 onward, until the end of the experiment. In summary, astaxanthin treatment caused a significant increase in expression of wound healing biomarkers Col1A1 and bFGF, but a significant decrease in expression of oxidative stress (ROS) biomarker iNOS (Figure 4).

\section{Discussion}

Significant accelerated healing effects of astaxanthin were observed on the first day after injury, during the inflammatory phase. This effect could be mediated either by suppression of the inflammation level or by acceleration of the inflammatory phase. Minimal inflammation is known to contribute to better wound healing. Astaxanthin may suppress this unfavorable condition through various mechanisms. First, this effect could be facilitated by balancing oxidative stress. While the production of ROS in the early phase is significantly higher than normal in order to defend against invading microorganisms and transmit intercellular signals supporting the process of inflammation, ${ }^{11,12}$ astaxanthin may be quenching and scavenging excessive ROS and RNS, which is consistent with our result which showed significantly decreased expression of iNOS, an oxidative stress marker, in the astaxanthintreated group. As a result, activation of the NF-kB pathway may be prevented, leading to reduced pro-inflammatory gene transcription and pro-inflammatory cytokine production. ${ }^{13,14}$ Second, astaxanthin may have an inhibitory effect on the expression of adhesion molecules. The main source of ROS during inflammation is NADPH oxidase in the plasma membrane of neutrophils and macrophages. It was found that traditional antioxidants suppress expression of adhesion molecules (ICAM-1, VCAM-1, E-selectin) and chemokines (IL-8) during inflammation. ${ }^{15,16}$ Astaxanthin may also inhibit expression of these molecules, leading to inhibition of inflammatory cell infiltration. ROS production in mitochondria was found to be involved in inflammatory signaling pathways, ${ }^{16}$ and the protective effects of astaxanthin may protect against

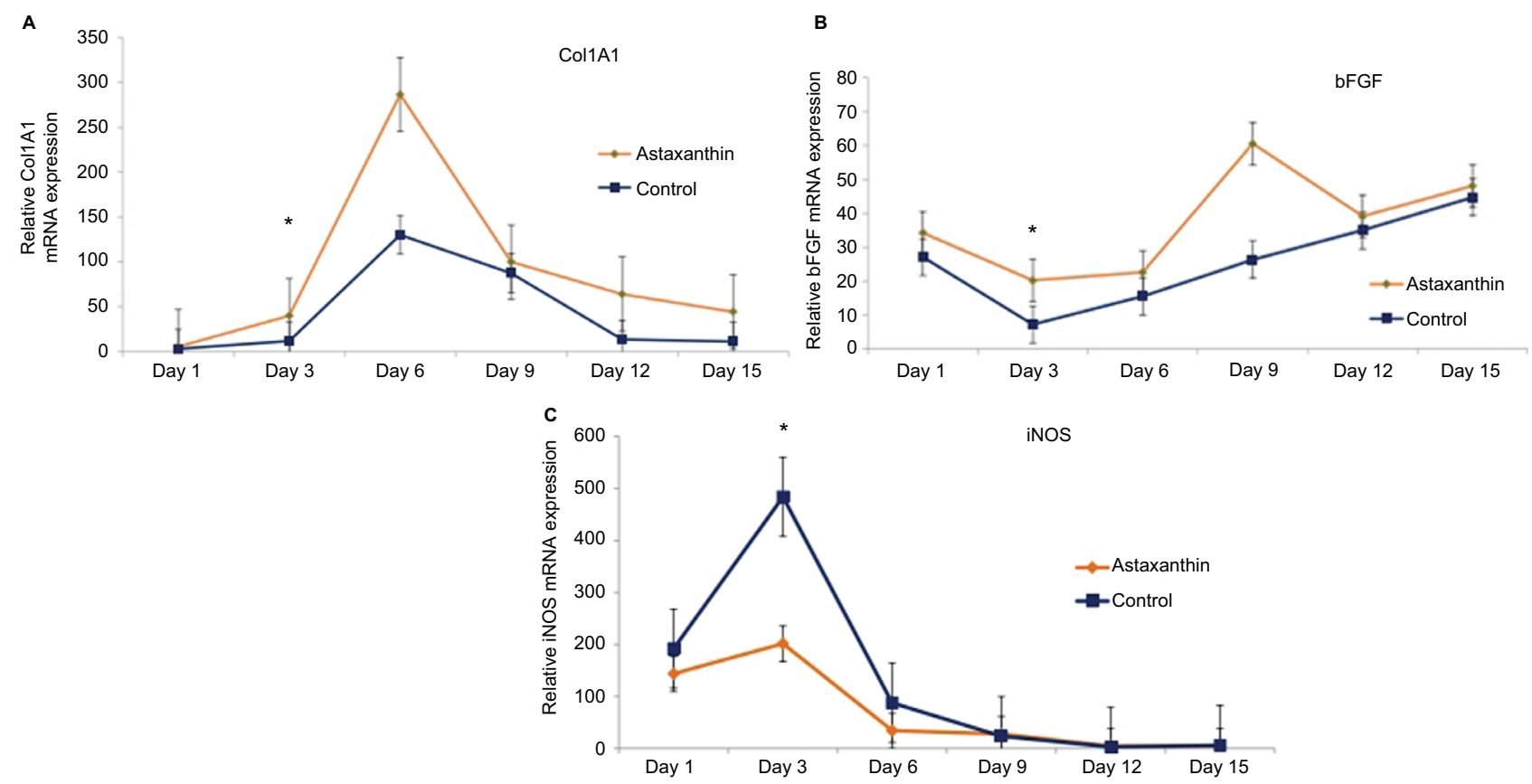

Figure 4 The mRNA expression of tissue-specific wound healing biomarkers.

Notes: Gene expression of wound healing and oxidative stress markers were analyzed using real-time polymerase chain reaction analysis of astaxanthin-treated mice and control mice, represented by the orange and the blue line, respectively. The (A-C) show the expression of CollAI, bFGF, and iNOS, respectively. The asterisk represents a significant difference in expression $(P<0.05)$ on the indicated day. Error bars indicate standard deviations. 
these intracellular oxidative molecules as well. ${ }^{5}$ In addition, its anticomplement activity may also be involved in the suppression of inflammation. ${ }^{17}$

The results during the proliferative and remodeling phases also indicate the significant potential of astaxanthin to reduce wound size throughout the closure process. This correlates with the increased expression of bFGF due to astaxanthin in our study. bFGF plays a significant role in granulation tissue formation, reepithelialization, matrix formation, and remodeling, ${ }^{18}$ which are the major events during proliferative and remodeling phases. In vitro studies have demonstrated that bFGF regulates the synthesis and deposition of various EMC components, increases keratinocyte motility during reepithelialization, ${ }^{19}$ promotes the migration of fibroblasts, and stimulates them to produce collagenase. The increased expression of bFGF mRNA in the astaxanthin-treated group during the early phase of wound healing may contribute to a significant acceleration of wound closure.

As mentioned previously, a low concentration of ROS is needed to initiate a normal repair process. Astaxanthin was shown to preserve that physiological function by redox regulation. For example, in host defense mechanisms, astaxanthin suppresses ROS and inflammatory cell infiltration. At the same time, it improves the capacity and ability of leukocytes to destroy pathogens. ${ }^{20}$ In angiogenesis, ROS signals regulate formation of new blood vessels. Astaxanthin may enhance the effect of ROS in activating physiological angiogenesis, and regulate ROS at an appropriate level, which is not harmful to endothelial cells. ${ }^{21}$ Additionally, it may also inhibit pathological angiogenesis in vascularized tumors by suppressing angiogenesis via the JAK2/STAT3 signaling pathway. ${ }^{22}$

Collagen is an essential component of the proliferative and remodeling phase, as it provides strength to the wound. ${ }^{23,24}$ In the early process of granulation tissue formation, fibroblasts produce collagen type 3 , which is later substituted with a stronger type 1 collagen during the maturation phase. Our data showed a significantly higher expression of Col1 A1 on day 6 after injury in the astaxanthin-treated group, compared to the control group. This is consistent with a study on vocal fold wound healing, ${ }^{25}$ which found that astaxanthin upregulates Col1A1 expression. Wound contraction is another important mechanism in the process of wound closure, especially in rodents. ${ }^{26}$ Astaxanthin probably accelerates wound contraction during the proliferative and maturation phase by enhancing the function of myofibroblasts, the cells that are differentiated from tissue fibroblasts, which play a key role in this process. Their smooth muscle features produce contractile forces between the wound edges and ECM. They also produce collagen matrix to form scar tissue, and release cytokines and growth factors contributing to the increasing rate of wound healing. ${ }^{27}$ However, in later stages, the myofibroblasts are removed from the normally healed wound; a persistent accumulation possibly results in hypertrophic scar or keloid. ${ }^{28}$ In our study, there was no visible scar observed at the end of the study in both groups. While increasing the contraction of the wound, astaxanthin may also reduce the chance of fibrosis by inducing apoptosis of the myofibroblast and/or suppressing TGF- $\beta .^{29,30}$

\section{Conclusion}

Topical treatment with astaxanthin extract appears to accelerate wound healing in full-thickness dermal wounds in mice. Future studies are likely to employ astaxanthin as a novel redox-based strategy to treat wounds in humans.

\section{Acknowledgment}

The authors gratefully acknowledge the financial support provided by Chulabhorn International College of Medicine, Thammasat University, contract no 11/2558.

\section{Disclosure}

The authors report no conflicts of interest in this work.

\section{References}

1. Braiman-Wiksman L, Solomonik I, Spira R, Tennenbaum T. Novel insights into wound healing sequence of events. Toxicol Pathol. 2007;35(6):767-779.

2. Roy S, Khanna S, Sen CK. Redox regulation of the VEGF signaling path and tissue vascularization: hydrogen peroxide, the common link between physical exercise and cutaneous wound healing. Free Radic Biol Med. 2008;44(2):180-192.

3. Schieber M, Chandel N. ROS function in redox signaling and oxidative stress. Curr Biol. 2014;24(10):R453-R462.

4. Ojha N, Roy S, He G, et al. Assessment of wound-site redox environment and the significance of Rac2 in cutaneous healing. Free Radic Biol Med. 2008;44(4):682-691.

5. auf dem Keller U, Kümin A, Braun S, Werner S. Reactive oxygen species and their detoxification in healing skin wounds. J Investig Dermatology Symp Proc. 2006;11(1):106-111.

6. Galeano M, Torre V, Deodato B, et al. Raxofelast, a hydrophilic vitamin E-like antioxidant, stimulates wound healing in genetically diabetic mice. Surgery. 2001;129(4):467-477.

7. Fitzmaurice SD, Sivamani RK, Isseroff RR. Antioxidant therapies for wound healing: a clinical guide to currently commercially available products. Skin Pharmacol Physiol. 2011;24(3):113-126.

8. Süntar I, Akkol EK, Nahar L, Sarker SD. Wound healing and antioxidant properties: do they coexist in plants? Free Radicals Antioxidants. 2012;2(2):1-7.

9. Nishida Y, Yamashita E, Miki W. Quenching activities of common hydrophilic and lipophilic antioxidants against singlet oxygen using chemiluminescence detection system. Carotenoid Sci. 2007;11:16-20.

10. Hussein G, Sankawa U, Goto H, Matsumoto K, Watanabe H. Astaxanthin, a carotenoid with potential in human health and nutrition. $J$ Nat Prod. 2006;69(3):443-449. 
11. Mizuta M, Hirano S, Ohno S, et al. Expression of reactive oxygen species during wound healing of vocal folds in a rat model. Ann Otol Rhinol Laryngol. 2012;121(12):804-810.

12. Lee SJ, Bai SK, Lee KS, et al. Astaxanthin inhibits nitric oxide production and inflammatory gene expression by suppressing I(kappa)B kinase-dependent NF-kappaB activation. Mol Cells. 2003;16(1):97-105.

13. Demianenko IA, Vasilieva TV, Domnina LV, et al. Novel mitochondriatargeted antioxidants, "Skulachev-Ion" derivatives, accelerate dermal wound healing in animals. Biochemistry (Mosc). 2010;75(3):274-280.

14. Lin SJ, Shyue SK, Hung YY, et al. Superoxide dismutase inhibits the expression of vascular cell adhesion molecule-1 and intracellular cell adhesion molecule- 1 induced by tumor necrosis factor-alpha in human endothelial cells through the JNK/p38 pathways. Arterioscler Thromb Vasc Biol. 2005;25(2):334-340.

15. Kim I, Moon SO, Park SK, Chae SW, Koh GY. Angiopoietin-1 reduces VEGF-stimulated leukocyte adhesion to endothelial cells by reducing ICAM-1, VCAM-1, and E-selectin expression. Circ Res. 2001;89(6):477-479.

16. Lowes DA, Thottakam BM, Webster NR, Murphy MP, Galley HF. The mitochondria-targeted antioxidant MitoQ protects against organ damage in a lipopolysaccharide-peptidoglycan model of sepsis. Free Radic Biol Med. 2008;45(11):1559-1565.

17. Lauver DA, Lockwood SF, Lucchesi BR. Disodium disuccinate astaxanthin (Cardax) attenuates complement activation and reduces myocardial injury following ischemia/reperfusion. J Pharmacol Exp Ther. 2005;314(2):686-692.

18. Barrientos S, Stojadinovic O, Golinko MS, Brem H, Tomic-Canic M. Growth factors and cytokines in wound healing. Wound Repair Regen. 2008;16(5):585-601.

19. Sogabe Y, Abe M, Yokoyama Y, Ishikawa O. Basic fibroblast growth factor stimulates human keratinocyte motility by Rac activation. Wound Repair Regen. 2006;14(4):457-462.
20. Macedo RC, Bolin AP, Marin DP, Otton R. Astaxanthin addition improves human neutrophils function: in vitro study. Eur J Nutr. 2010;49(8):447-457.

21. Xu Y, Zhang J, Jiang W, Zhang S. Astaxanthin induces angiogenesis through $\mathrm{Wnt} / \beta$-catenin signaling pathway. Phytomedicine. 2015;22(7-8):744-751.

22. Kowshik J, Baba AB, Giri H, Deepak Reddy G, Dixit M, Nagini S. Astaxanthin inhibits JAK/STAT-3 signaling to abrogate cell proliferation, invasion and angiogenesis in a hamster model of oral cancer. PLoS One. 2014;9(10):e109114.

23. Carlson MA, Longaker MT. The fibroblast-populated collagen matrix as a model of wound healing: a review of the evidence. Wound Repair Regen. 2004;12(2):134-147.

24. Werner S, Krieg T, Smola H. Keratinocyte-fibroblast interactions in wound healing. J Invest Dermatol. 2007;127(5):998-1008.

25. Mizuta M, Hirano S, Hiwatashi N, et al. Effect of astaxanthin on vocal fold wound healing. Laryngoscope. 2014;124(1):E1-E7.

26. Park SA, Covert J, Teixeira L, et al. Importance of defining experimental conditions in a mouse excisional wound model. Wound Repair Regen. 2015;23(2):251-261.

27. Hinz B, Phan SH, Thannickal VJ, Galli A, Bochaton-Piallat ML, Gabbiani G. The myofibroblast: one function, multiple origins. Am J Pathol. 2007;170(6):1807-1816.

28. Hinz B. Formation and function of the myofibroblast during tissue repair. J Invest Dermatol. 2007;127(3):526-537.

29. Wang M, Zhang J, Song X, Liu W, Zhang L, Wang X, Lv C. Astaxanthin ameliorates lung fibrosis in vivo and in vitro by preventing transdifferentiation, inhibiting proliferation, and promoting apoptosis of activated cells. Food Chem Toxicol. 2013;56:450-458.

30. Shen M, Chen K, Lu J, et al. Protective effect of astaxanthin on liver fibrosis through modulation of TGF- $\beta 1$ expression and autophagy. Mediators Inflamm. 2014;2014:954502.
Clinical, Cosmetic and Investigational Dermatology

\section{Publish your work in this journal}

Clinical, Cosmetic and Investigational Dermatology is an international, peer-reviewed, open access, online journal that focuses on the latest clinical and experimental research in all aspects of skin disease and cosmetic interventions. This journal is included on PubMed. The manuscript management system is completely online

\section{Dovepress}

and includes a very quick and fair peer-review system, which is all easy to use. Visit http://www.dovepress.com/testimonials.php to read real quotes from published authors 